\title{
Multispectral imaging of organ viability during uterine transplantation surgery in rabbits and sheep
}

\author{
Neil T. Clancy \\ Srdjan Saso \\ Danail Stoyanov \\ Vincent Sauvage \\ David J. Corless \\ Michael Boyd \\ David E. Noakes \\ Meen-Yau Thum \\ Sadaf Ghaem-Maghami \\ James Richard Smith \\ Daniel S. Elson
}




\title{
Multispectral imaging of organ viability during uterine transplantation surgery in rabbits and sheep
}

\author{
Neil T. Clancy, ${ }^{\mathrm{a}, \mathrm{b}, *}$ Srdjan Saso, ${ }^{\mathrm{c}}$ Danail Stoyanov ${ }^{\mathrm{d}}{ }^{\text {Vincent Sauvage, }},{ }^{\mathrm{a}, \mathrm{b}}$ David J. Corless, ${ }^{\mathrm{e}}$ Michael Boyd, ${ }^{\mathrm{f}}$ \\ David E. Noakes, ${ }^{f}$ Meen-Yau Thum, ${ }^{g}$ Sadaf Ghaem-Maghami,${ }^{c}$ James Richard Smith, ${ }^{\mathrm{h}}$ and Daniel S. Elson ${ }^{\mathrm{a}, \mathrm{b}}$ \\ amperial College London, Institute of Global Health Innovation, Hamlyn Centre for Robotic Surgery, London SW7 2AZ, United Kingdom \\ ${ }^{b}$ Imperial College London, Division of Surgery, Department of Surgery and Cancer, London SW7 2AZ, United Kingdom \\ 'Imperial College London, Hammersmith Hospital Campus, Department of Surgery and Cancer, Institute of Reproductive and \\ Developmental Biology, London W12 0NN, United Kingdom \\ dUniversity College London, Department of Computer Science, Centre for Medical Image Computing, London WC1E 6BT, United Kingdom \\ eLeighton Hospital, Mid Cheshire Hospitals NHS Foundation Trust, Department of Surgery, Crewe CW1 4QJ, United Kingdom \\ ${ }^{\mathrm{f}}$ Royal Veterinary College, Biological Services Unit, London NW1 OTU, United Kingdom \\ ${ }^{9}$ The Lister Hospital, London SW1W 8RH, United Kingdom \\ 'Imperial College London, Hammersmith Hospital Campus, Queen Charlotte's Hospital, West London Gynaecological Cancer Centre, \\ London W12 0NN, United Kingdom
}

\begin{abstract}
Uterine transplantation surgery (UTx) has been proposed as a treatment for permanent absolute uterine factor infertility (AUFI) in the case of the congenital absence or surgical removal of the uterus. Successful surgical attachment of the organ and its associated vasculature is essential for the organ's reperfusion and long-term viability. Spectral imaging techniques have demonstrated the potential for the measurement of hemodynamics in medical applications. These involve the measurement of reflectance spectra by acquiring images of the tissue in different wavebands. Measures of tissue constituents at each pixel can then be extracted from these spectra through modeling of the light-tissue interaction. A multispectral imaging (MSI) laparoscope was used in sheep and rabbit UTx models to study short- and long-term changes in oxygen saturation following surgery. The whole organ was imaged in the donor and recipient animals in parallel with point measurements from a pulse oximeter. Imaging results confirmed the re-establishment of adequate perfusion in the transplanted organ after surgery. Cornual oxygenation trends measured with MSI are consistent with pulse oximeter readings, showing decreased $\mathrm{StO}_{2}$ immediately after anastomosis of the blood vessels. Long-term results show recovery of $\mathrm{StO}_{2}$ to preoperative levels. ( ) The Authors. Published by SPIE under a Creative Commons Attribution 3.0 Unported License. Distribution or reproduction of this work in whole or in part requires full attribution of the original publication, including its DOI. [DOI: 10.1117/1.JBO.21.10.106006]
\end{abstract}

Keywords: uterine transplantation; multispectral imaging; oxygen saturation; surgical imaging.

Paper 160418PR received Jun. 16, 2016; accepted for publication Oct. 5, 2016; published online Oct. 27, 2016.

\section{Introduction}

Recently published results by Brännström et al. ${ }^{1}$ have demonstrated the feasibility of uterine transplantation surgery (UTx) to offer the potential for pregnancy to women without a womb. An important predictor of the success of the transplant procedure is the degree to which perfusion is restored in the organ. An optical method of assessing the whole organ's health is described in this paper within the context of an animal study.

\subsection{Uterine Transplantation Surgery}

Absolute uterine factor infertility (AUFI) renders women unconditionally infertile due to the absence of the uterus (premenopausal hysterectomy or congenital reasons). ${ }^{2}$ UTx has been proposed as a potential solution in such cases. A large body of surgical research in animal transplant models, ${ }^{3}$ in addition to experience in fertility-sparing oncological surgery, ${ }^{4}$ has resulted in the development of surgical techniques that recently led to the first successful UTx in humans. ${ }^{1}$

Reperfusion of the organ, through connection of its major blood vessels to the recipient's vasculature, is necessary to ensure long-term viability of the uterus. This is particularly

*Address all correspondence to: Neil T. Clancy, E-mail: n.clancy@imperial.ac .uk important as the organ is deprived of oxygenated blood during periods of vascular clamping ("warm" ischaemia) or while preserved outside the body during surgery ("cold" ischaemia). Ischemia-reperfusion injury to the parenchyma and microcirculation may occur during these periods. ${ }^{2}$

In previous work by our group, a pulse oximeter probe was used to measure oxygen saturation and "perfusion index" (a relative measurement of blood volume) by attaching it directly to the organ..$^{5}$ Although this method had the advantage of being readily available, it suffered from that fact that it only reflected arterial oxygenation and returned just single-point measurements.

\subsection{Optical Imaging of Hemodynamics}

Recently published results from our group have shown the feasibility of intraoperative visualization of oxygen saturation using a multispectral imaging (MSI) laparoscope. ${ }^{6}$ This technique, validated in a porcine abdominal procedure, was demonstrated to be capable of following changes in oxygenation across a wide range (30\% to $100 \%$ ), as well as being sensitive to the spatial heterogeneity of the tissue's vasculature. Studies by other researchers have also demonstrated the effectiveness of this approach for assessing perfusion of the retina, ${ }^{7}$ brain, ${ }^{8}$ and kidneys. ${ }^{9}$ Spectral reflectance imaging has also been used to investigate cold and warm ischaemia-reperfusion injury on the kidneys. ${ }^{10}$ 
In this paper, we describe the use of our laparoscopic MSI system to assess changes in uterine oxygen saturation during transplant surgery on rabbit and sheep models. The system incorporates a standard surgical white light source and a liquid crystal tuneable filter (LCTF) to provide spectral selection. Results were obtained in parallel with pulse oximeter point measurements in short- and long-term survival studies. Detailed descriptions of the surgical method and results have been published separately. ${ }^{11,12}$ These measurements enable intraoperative quantitative measures of perfusion to be recorded along with spatial information, at different time points in addition to subjective observations. The results of this study help to identify typical trends in organ oxygen saturation that may occur during surgery and recovery, with reference to a baseline.

\section{Materials and Methodology}

All animal experiments described in this paper were conducted under UK Home Office licenses (70/7508 and 70/6927). Full details of the surgical procedures may be found in separate papers relating to the sheep ${ }^{11}$ and rabbit operations. ${ }^{12}$

\subsection{Multispectral Imaging Laparoscope}

A laparoscopic MSI system developed by our group, and characterized and validated in a separate publication, ${ }^{6}$ has been used in the previous pilot studies of uterine transplantation procedures. ${ }^{13,14}$ The clinical imaging system comprises a 30-deg laparoscope (Karl Storz GmbH, Tuttlingen, Germany), LCTF (Varispec, Cri, Inc.), and monochrome camera (DCU223M, Thorlabs Ltd., UK). A surgical xenon light source (xenon 300, Karl Storz GmbH, Tuttlingen, Germany) was used for illumination. The laparoscope was secured to a flexible mounting arm and the whole system placed on a trolley that could be conveniently positioned at the bedside for measurements. A data cube of 13 images (500 to $620 \mathrm{~nm}$ ) was acquired to generate reflected intensity spectra of the tissue at each spatial location. Exposure times of $\sim 200 \mathrm{~ms} /$ image were required, resulting in a total acquisition time of $\sim 3.2 \mathrm{~s}$. A preprocessing image registration step using custom-written feature-tracking software ${ }^{15,16}$ was carried out to compensate for misalignments caused by breathing and peristalsis motion during this time. The spectral sensitivity of the optical system was corrected for using a white reflectance target (Spectralon, Labsphere, Inc.) before converting the data to absorbance spectra. Figure 1 shows the laparoscope in position during imaging of the rabbit uterus.

Relative concentrations of oxygenated $\left(\mathrm{HbO}_{2}\right)$ and deoxygenated hemoglobin $(\mathrm{Hb})$ were determined using linear least squares regression ${ }^{6}$ of the experimental data to the known pure component spectra. ${ }^{17}$ The sum of the concentrations (total hemoglobin; $\mathrm{Hbt}$ ) was calculated, along with the concentration of $\mathrm{HbO}_{2}$ expressed as a percentage of $\mathrm{Hbt}$ (oxygen saturation; $\mathrm{StO}_{2}$ ). This approach is subject to the assumption that oxy- and deoxyhemoglobin are the only optical absorbers in the field-ofview, and that scattering and penetration depth are approximately constant across the spectrum. Pixel locations with a poor match $\left(r^{2}<0.9\right)$ between experimental and model absorbance curves were discarded. All data processing was conducted offline using MATLAB (The Math Works, Inc.).

\subsection{Intraoperative Imaging}

Uterine oxygenation was measured during short-term (sheep) and long-term (rabbit) animal UTx viability studies. The sheep

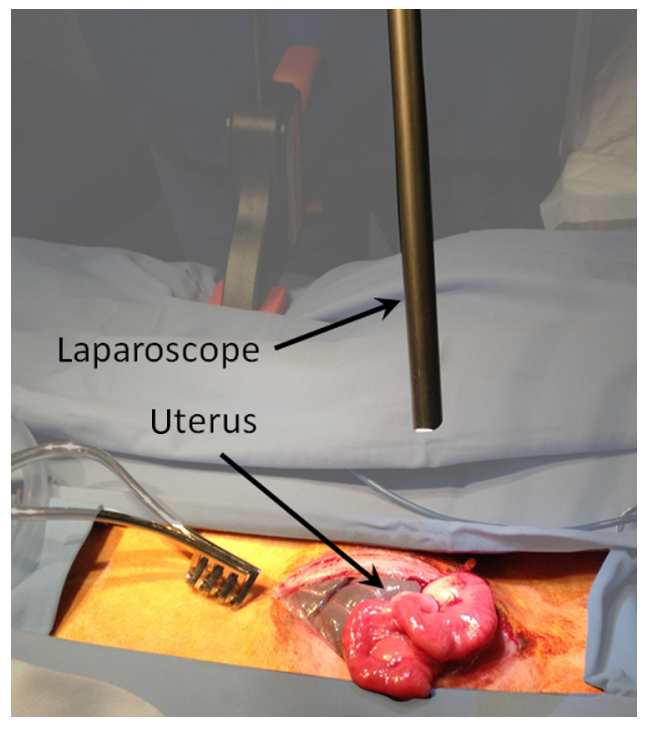

Fig. 1 Intraoperative MSI during rabbit UTx. The distance between the laparoscope tip and the organ is $\sim 10 \mathrm{~cm}$ and the field-of-view of the system is $\sim 6 \mathrm{~cm}$ in diameter.

( $\sim 60 \mathrm{~kg})$ study was conducted as an autotransplant under terminal anaesthesia, with the uterus removed, stored externally temporarily, and replaced into the same animal. New Zealand white rabbits $(\sim 4 \mathrm{~kg})$ were used in a series of allotransplant procedures, where the uterus of a donor animal was transplanted to a recipient animal. The recipient subsequently recovered following surgery.

The transplant procedure involved removal of the uterus and its blood supply en bloc, in a microvascular patch, followed by flushing of the blood vessels with saline and a preservation solution (Custodiol HTK, Dr. Franz Köhler Chemie GmbH, Germany). The organ was stored on ice in a bath of the same preservation solution while the recipient was prepared. During implantation, the cornua and vagina were also attached to provide mechanical stability for the organ. Reconnection of the blood supply (anastomosis) was achieved either through an aorta-aorta/inferior vena cava (IVC)-IVC anastomosis (rabbit) or internal/external iliac anastomosis (sheep), as illustrated in Fig. 2.

Oxygenation measurements were made of the uterus in its native condition, during organ retrieval surgery in the donor, and immediately after implantation and reperfusion in the recipient. In the case of the allotransplant study, the recipient's own uterus was also measured before a hysterectomy was performed. A pulse oximeter (Datex-Ohmeda 3600P; DatexOhmeda, Colorado) was used to measure oxygen saturation and perfusion index (PI) at the medial and lateral aspects of the right and left cornua (Fig. 2). These measurements were followed by MSI acquisitions of the entire uterus. Three MSI measurements were made at each site to reduce the risk of random error. Mean $\mathrm{StO}_{2}$ was calculated in the regions-of-interest within the images that corresponded to the pulse oximeter measurement sites.

\section{Results}

The short-term (autotransplant) and long-term (allotransplant) MSI results are presented below and show reconstructed color images and $\mathrm{StO}_{2}$ overlays. Imaging was completed in a total of seven rabbits and five sheep. Each transplant procedure is referred to by a UTx number (rabbits: 3 to 9; sheep: 1 to 5) 


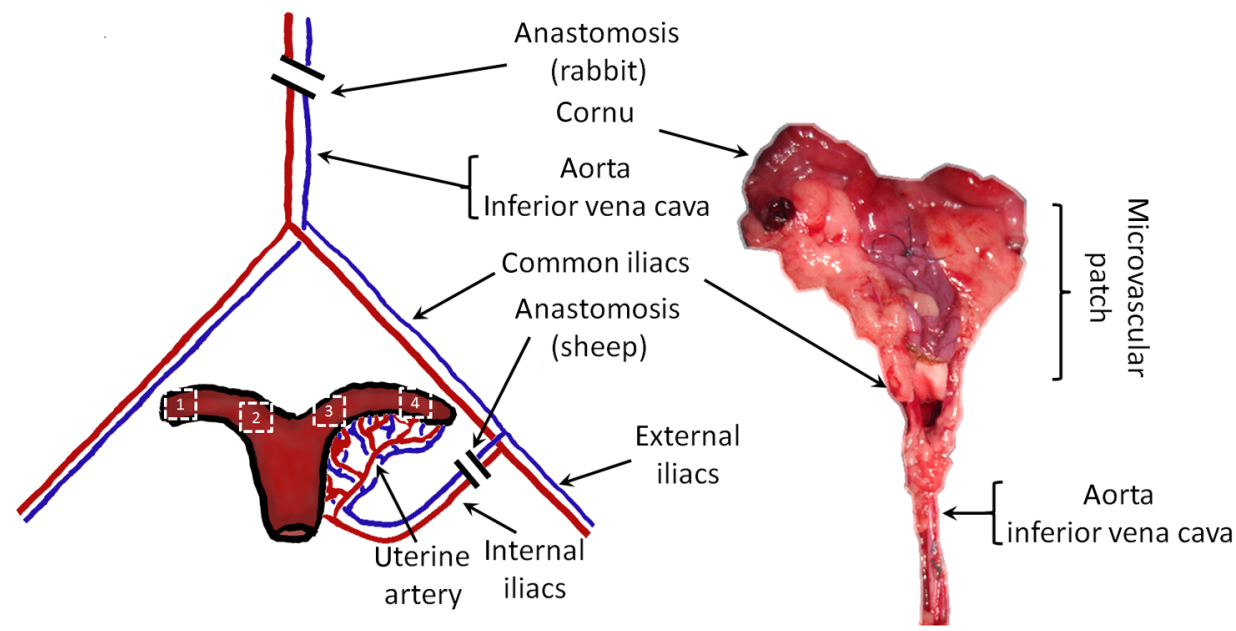

Fig. 2 Schematic showing the harvested uterus, its major supplying blood vessels, and the points of anastomosis for the rabbit and sheep transplants. The numbered regions of interest indicate measurement points for oxygen saturation on the medial and lateral parts of the uterine cornua. The photograph on the right shows a rabbit uterus immediately prior to removal from the donor animal. The aorta and inferior vena cava appear below the organ following dissection of the microvascular patch.

corresponding to the results described in our surgical papers. ${ }^{11,12}$ No imaging was performed in rabbit UTx 1 or 2.

\subsection{Sheep Study (Autotransplantation)}

Color and processed MSI data from the sheep transplants are shown in Fig. 3. On appearance alone, the uterus, in its native state, appears bright red/pink as it is well-perfused with oxygenated blood. The transplanted uterus ("graft"), while also red, is not as saturated as in the native state. The processed MSI data also support this, with the bright red and yellow shades indicating relatively high oxygenation in both cases. Areas that could not be adequately described by the absorbance model are seen as "gaps" in the processed images. This is particularly evident in
Donor

$$
\text { UTx }
$$

1

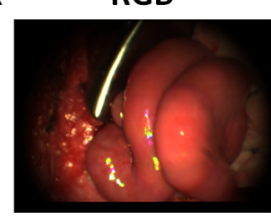

2

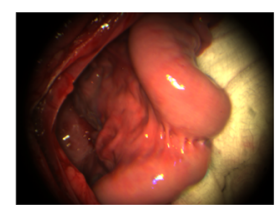

3

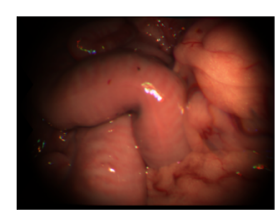

4
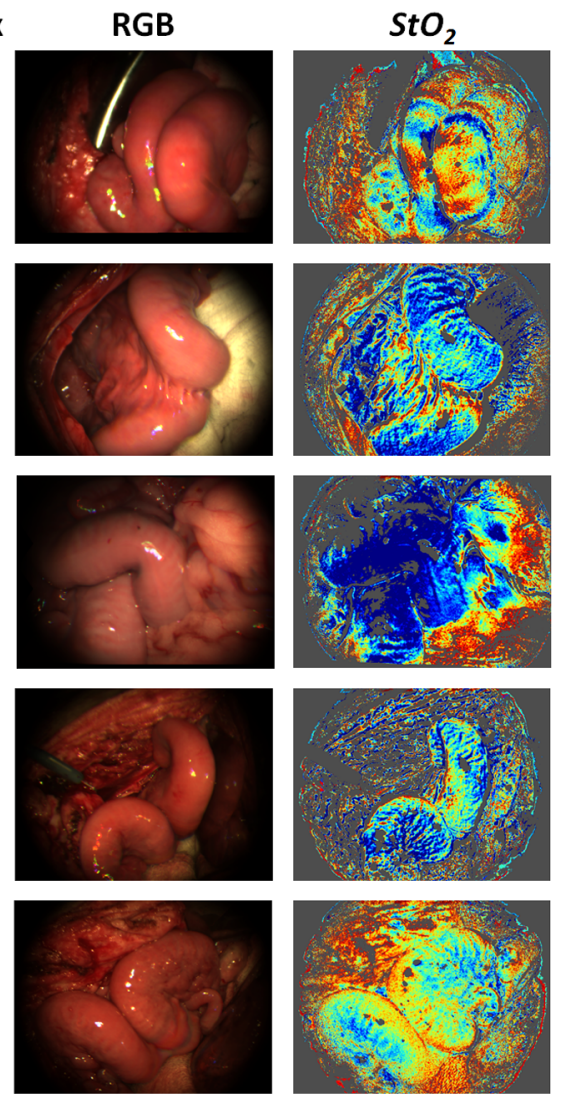

\section{Graft}
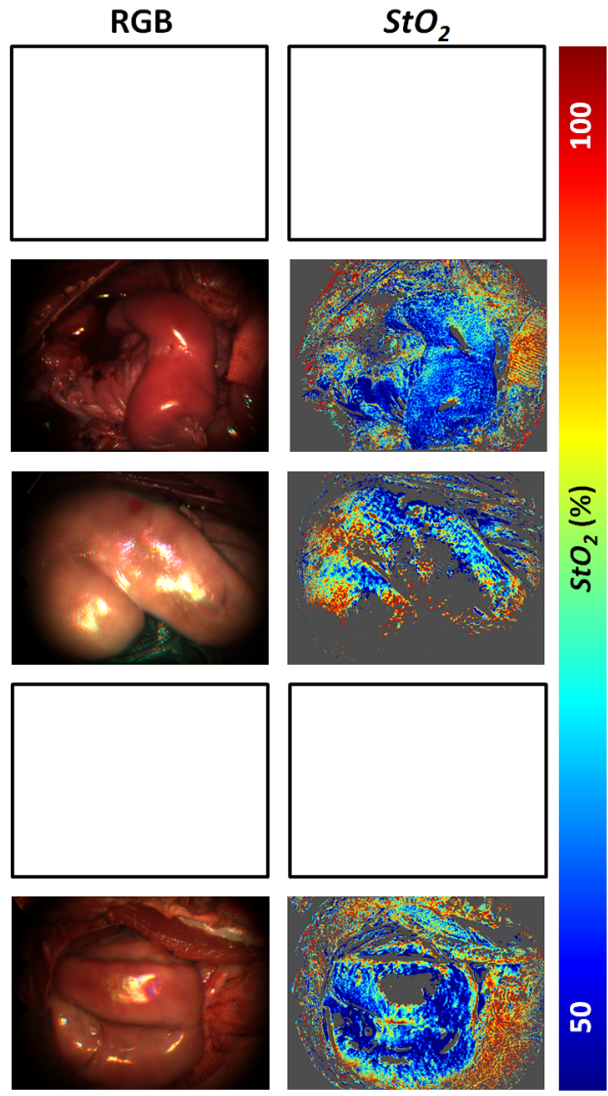

Fig. 3 Complete sheep imaging results showing the uterus in the donor, prior to harvesting, and the transplanted graft. UTx 1 and 4 were abandoned during implantation. 


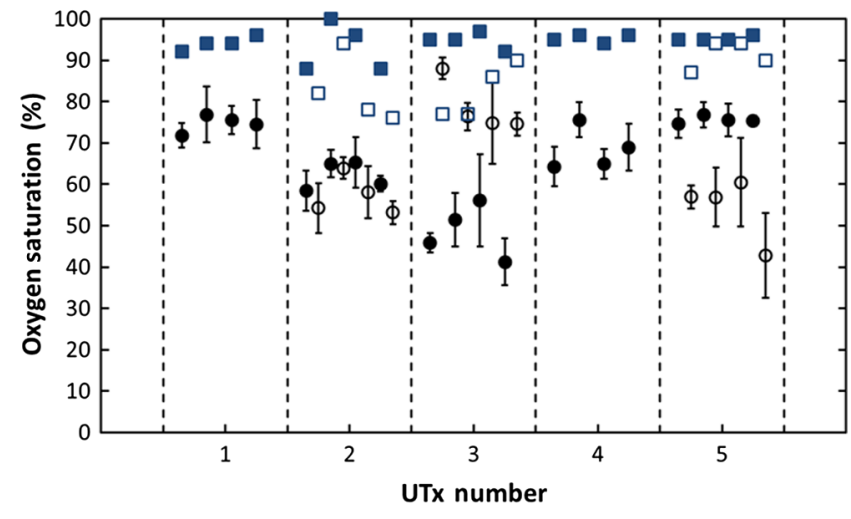

- Donor (MSI) O Graft (MSI) a Donor (Pulse Ox.) $\quad$ aGraft (Pulse Ox.)

Fig. 4 Mean cornual $\mathrm{StO}_{2}$, plotted against sheep autotransplant number, for the four regions-of-interest in the donor uterus and transplanted graft. The error bars represent the standard deviation of repeated measures of the same organ. Pulse oximeter measurements $\left(\mathrm{SpO}_{2}\right)$ for the same regions of the organ are plotted alongside the MSI results. regions where specular highlights are present, such as those visible in Fig. 3.

The quantitative results are shown in Fig. 4. Transplant numbers 1 and 4 had to be abandoned due to intraoperative complications. ${ }^{11}$ The rest were completed as planned.

Baseline (donor) arterial oxygen saturation values recorded by the pulse oximeter are consistently high in all animals, varying from $88 \%$ to $100 \%$ (mean $=94 \%$ ). Tissue oxygen saturation measured by the MSI device is lower, varying from $41 \%$ to $77 \%$ (mean $=66 \%)$. Intraorgan variability is visible in the mean values in the four ROIs of each case, whereas interorgan variability in $\mathrm{StO}_{2}$ is evident through comparison of the donor and recipient values across all procedures. In the completed procedures (UTx 2, 3, 5), lower oxygen saturation values were recorded in the transplanted graft by both methods, with the exception of the MSI results for UTx 3.

\subsection{Rabbit Study (Allotransplant)}

Macroscopically, the appearance of the rabbit uteri, shown in Fig. 5 immediately pre- and posttransplant, is similar to that of the sheep. The native organ in the donor and recipient (prehysterectomy) appears bright and well-perfused, while appearing

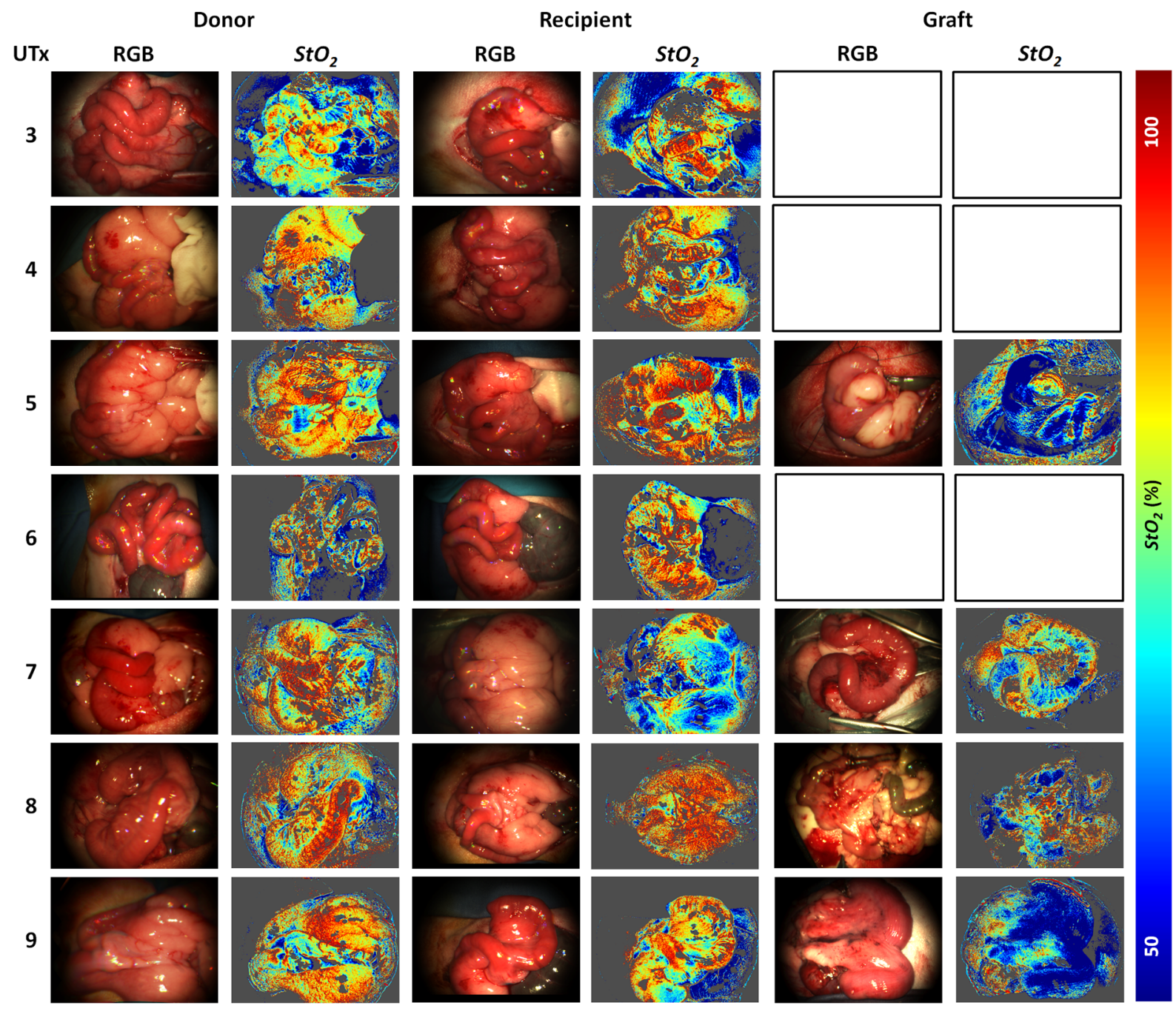

Fig. 5 Complete rabbit MSI results showing images for the donor, the recipient's native uterus and the transplanted graft. Pixels with $r^{2}<0.9$ are grayed out. 


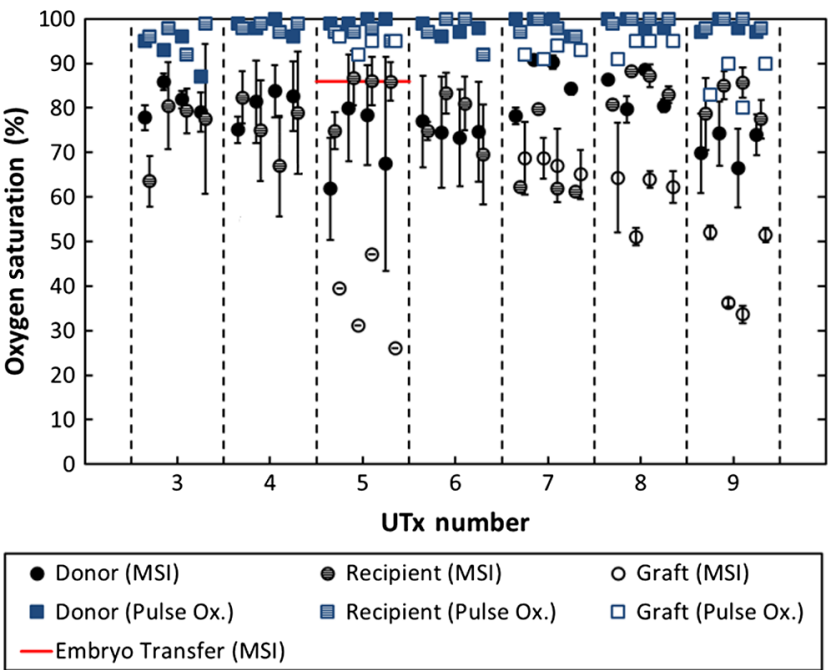

Fig. 6 Mean cornual oxygen saturation, plotted against transplant number, in the regions-of-interest in the donor animal, the recipient (native uterus), and the transplanted graft. Identification of the ROIs at embryo transfer (UTX 5) was not possible, due to adhesions (see Fig. 7), so mean $\mathrm{StO}_{2}$ for the entire organ is shown instead. Error bars represent the standard deviation of repeated measurements on the same organ. Pulse oximeter measurements for the corresponding regions are included on the same plot.

less-so in the transplanted organ. The quantitative MSI results follow the same trend. The resolution achieved with the MSI laparoscope is $\sim 0.3 \mathrm{~mm} /$ pixel at a working distance of $10 \mathrm{~cm}$.

Quantitative results for the native (donor and recipient) and transplanted uterus are shown in Fig. 6. There were also intraoperative complications in some of the test subjects, and the rabbits in UTx 3, 4, and 6 died during anastomosis of the donor vasculature to that of the recipient. ${ }^{12}$ In these cases, no oximetry measurements of the graft are presented.

Baseline oxygen saturation levels in the uterus (both the donors' and recipients' native organs) are high in all animals, varying from $87 \%$ to $100 \%($ mean $=98 \%)$. Similar to the sheep results, inter- and intraorgan variability can be seen in the scatter of $\mathrm{StO}_{2}$ values in each ROI and between different animals. For a particular organ, the transplanted uterus shows a consistently lower $\mathrm{StO}_{2}$ immediately posttransplantation than the native organ across the entire dataset. This is also borne out by the pulse oximeter readings for UTx $5,7,8$, and 9 . The MSI results are lower than the pulse oximeter in every case.
The long-term survivor (UTx 5) was imaged 90 days posttransplant during the surgical transfer of embryos to the transplant recipient's uterus. Mean $\mathrm{StO}_{2}$ in the organ recovered to $86 \% \pm 1 \%$ from the immediate postoperative value of $47 \%$, which is also higher than the original baseline reading of $80 \% \pm$ $12 \%$ (medial aspect). The color and MSI images of the organ, shown in Fig. 7, indicate the changes that the organ has undergone in the intervening period. A significant number of postoperative adhesions have hidden the uterus's distinctive shape, but it is clear that perfusion has been fully re-established.

\section{Discussion}

Donor uteri, in both rabbit and sheep procedures, were wellperfused and oxygenated as evident from the $\mathrm{StO}_{2}$ images. Mean $\mathrm{StO}_{2}$ values from the regions-of-interest varied from $62 \%$ to $91 \%$ (mean $=78 \%$ ) in the rabbits, and from $41 \%$ to $77 \%$ (mean $=66 \%)$ in the sheep. Reperfusion of the organ was confirmed by detection of a pulsatile signal with the pulse oximeter and by the MSI images, which displayed a clear hemoglobin signal. Tissue oxygen saturation in the transplanted graft, as measured by the imager, was markedly decreased in all cases (with the exception of sheep UTx 3) compared to the native state. A paired $t$-test returns a significant difference between the $\mathrm{StO}_{2}$ values pre- and post-UTx $\left(p=2.3 \times 10^{-8}\right)$ in the rabbit. This may be an effect related to the fact that even though oxygenated blood was flowing through the organ's main vessels many microvessels within the tissue had still not dilated fully or been perfused with oxygenated blood. Thus, the more superficial tissue with lower oxygen content may have a comparatively stronger effect on the final $\mathrm{StO}_{2}$ value recorded by the imager, which integrates the signal from both venous and arterial blood.

The MSI laparoscope uses the 500 to $620-\mathrm{nm}$ range to estimate $\mathrm{StO}_{2}$ due to the large difference in shape between the oxy- and deoxyhemoglobin absorption curves in this region. As mentioned in our previous work, ${ }^{6}$ this is under the assumption that each wavelength of detected light interrogates an equal volume of tissue. However, using values for whole blood, ${ }^{18}$ it can be seen that the effective attenuation coefficient $\left(\mu_{\text {eff }}=\sqrt{3 \mu_{a}\left(\mu_{a}+\mu_{s}^{\prime}\right)}\right)$, which influences penetration depth, varies by $48 \%$ across this range. This is similar to other similar MSI processing examples in the literature, ${ }^{19-21}$ and is a necessary balance among pathlength assumptions, sensitivity to $\mathrm{StO}_{2}$ changes, quality of fit, and simplicity of computational implementation for imaging. It was found that the fitting range used here achieved better fit quality $\left(r^{2}\right)$ than a slightly narrower
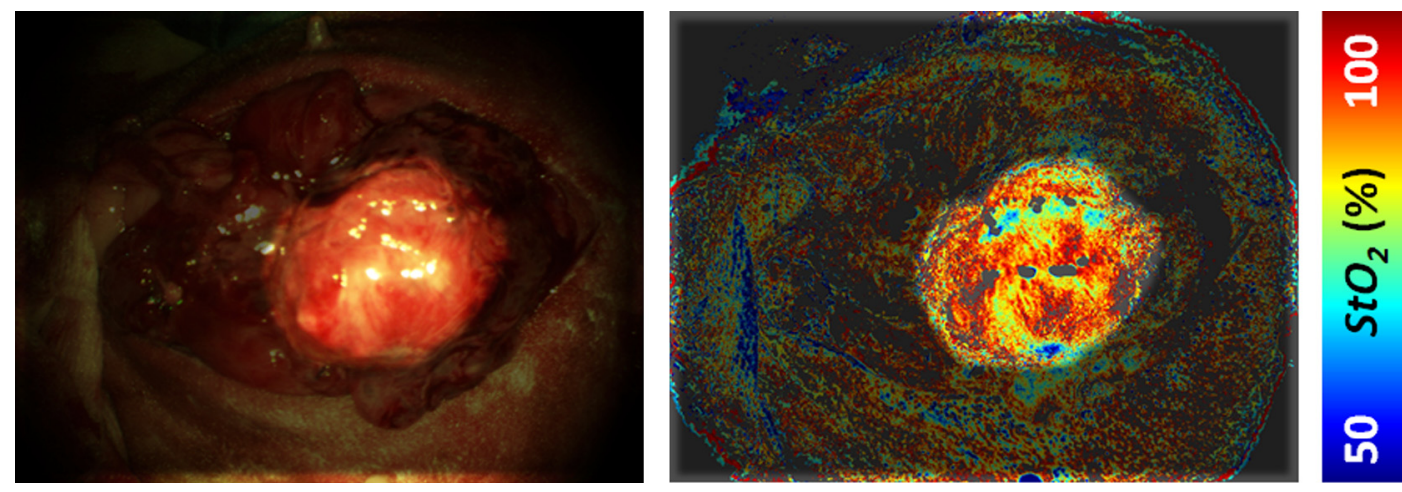

Fig. 7 Long-term survival and embryo transfer. Rabbit test subject imaged 90 days after transplantation, during the embryo transfer procedure. The uterus, highlighted, has lost its distinctive shape due to adhesions. Background tissue has been manually masked out for clarity. 
range (500 to $600 \mathrm{~nm}),{ }^{19}$ whereas $\mathrm{StO}_{2}$ was unaffected. Reducing the range further resulted in ever decreasing $r^{2}$ values, with $35 \%$ more pixels falling below the $r^{2}$ threshold when the 520 to $580 \mathrm{~nm}$ range is used to perform the regression.

The pulse oximeter also showed a statistically significant decrease in cornual oxygen saturation post-UTx (rabbit: $p=$ $5.5 \times 10^{-6}$; sheep: $p=5.8 \times 10^{-4}$ ), although the absolute values still remained higher than the MSI results. This is expected as the device uses arterial pulsations to measure the degree of optical absorption, and is thus principally reflective of arterial oxygen saturation. There was no significant difference in $\mathrm{StO}_{2}$ or $\mathrm{SpO}_{2}$ between the donor and recipient (native uterus) in the rabbit procedures ( $p=0.73$ and $p=0.83$, respectively). There was a significant difference in PI between donor and graft in the rabbit procedures $(p=0.02)$, but not in the sheep procedures $(p=0.32)$.

Although both MSI and pulse oximetry detected a similar trend in the detection of a drop in oxygenation after transplantation, there was no statistically significant correlation between the absolute values of $\mathrm{StO}_{2}$ and $\mathrm{SpO}_{2}$. The main reason for this is most likely due to the significant differences between the two modalities in how the signal is acquired. MSI uses diffuse reflectance, integrating the signal from all blood (arterial and venous) within the top 2 to $3 \mathrm{~mm}$ of the tissue. The pulse oximeter, on the other hand, operates in transmission mode, measuring the signal from arterial blood in the uterine cornua between the jaws of the device.

In the long-term surviving rabbit (UTx 5), $\mathrm{StO}_{2}$ was measured at values close to that of baseline levels, indicating recovery of the microvasculature and confirming full reperfusion of the organ. The presence of adhesions meant that pulse oximetry was not possible.

\section{Conclusions}

MSI has been used for the first time to visualize organ oxygen saturation during UTx in rabbit and sheep models. The technique enabled noncontact quantitative assessment of the changes in uterine $\mathrm{StO}_{2}$ and blood volume. Pseudocolor maps allowed visualization of the spatial distribution of oxygenated blood, whereas regions-of-interest could also be selected for numerical comparison of $\mathrm{StO}_{2}$ at various stages in the transplant process.

MSI allows monitoring of organ perfusion during transplant surgery by quantitatively imaging tissue oxygen saturation. Although a number of commercial point probes exist (e.g., $\mathrm{O} 2 \mathrm{C}$; LEA Medizintechnik $\mathrm{GmbH}$, Germany; Intra. Ox; ViOptix, Inc.), there is currently no established method to perform $\mathrm{StO}_{2}$ imaging clinically, and the results presented here show its potential for use in future human studies. The device was capable of indicating restoration of perfusion following anastomosis of the organ's vasculature, as well as long-term recovery postoperatively. Neither the sheep nor the rabbit procedures described in this paper demonstrated any postoperative complications that could be directly linked to insufficient $\mathrm{StO}_{2}$. However, the consistently high $\mathrm{StO}_{2}$ values in the donor and recipient animals prior to surgery served as a useful baseline from which to track longitudinal changes. Future preclinical organ imaging studies, with controlled levels of perfusion, will help to quantify the ability of intraoperative $\mathrm{StO}_{2}$ as a predictor of tissue viability.

The main technical improvements needed to make this technique more suitable for clinical use involve reduction of the number of wavebands required for the $\mathrm{StO}_{2}$ calculation to increase acquisition speed, and development of more computationally efficient, parallel-processing methods. To this end, our group and collaborators have been investigating data reduction techniques, ${ }^{22}$ use of a fast filter wheel MSI camera, ${ }^{23}$ and implementation of machine learning algorithms. ${ }^{24}$ The imaging hardware is compatible with existing sterilizable medical instruments such as laparoscopes and rigid cystoscopes, meaning that incorporation of MSI into human UTx studies is feasible. It is hoped that $\mathrm{StO}_{2}$ measurements intraoperatively and later, transvaginally, may help to quantify the health of the organ, track its recovery after surgery, and possibly indicate the onset of any rejection episode. This type of imaging is of particular importance as some of the reported human UTx failures have been strongly linked with incomplete reperfusion of the uterus. ${ }^{25,26}$

\section{Acknowledgments}

Funding for this work was provided by ERC under Grant No. 242991, UK EPSRC Grant Nos. EP/E06342X/1 and DT/ E011101/1, and Uterine Transplant UK Charity (No. 1138559). Neil Clancy gratefully acknowledges the financial support of an Imperial College Junior Research Fellowship. The authors would like to thank the Royal Veterinary College for their assistance with surgical arrangements. We acknowledge David Harris-Birtill for help with data acquisition and photography. The surgeries described in this paper were carried out under Home Office project license numbers 70/7508 and 70/6927.

\section{References}

1. M. Brännström et al., "Livebirth after uterus transplantation," Lancet 385(9968), 607-616 (2015).

2. M. Brännström, C. A. Wranning, and A. Altchek, "Experimental uterus transplantation," Hum. Reprod. Update 16(3), 329-345 (2010).

3. M. Brännström et al., "Uterus transplantation: animal research and human possibilities," Fertil. Steril. 97(6), 1269-1276 (2012).

4. G. Del Priore et al., "Uterine transplantation, abdominal trachelectomy, and other reproductive options for cancer patients," Ann. N. Y. Acad. Sci. 943, 287-295 (2001).

5. P. Moxey et al., "Pulse oximetry and perfusion index measurement to assess uterine perfusion and viability," Int. Surg. 91(4), 223-227 (2006).

6. N. T. Clancy et al., "Intraoperative measurement of bowel oxygen saturation using a multispectral imaging laparoscope," Biomed. Opt. Express 6(10), 4179-4190 (2015).

7. J. C. Ramella-Roman and S. A. Mathews, "Spectroscopic measurements of oxygen saturation in the retina," IEEE Sel. Top. Quantum Electron. 13(6), 1697-1703 (2007).

8. D. Stoyanov, A. Rayshubskiy, and E. Hillman, "Robust registration of multispectral images of the cortical surface in neurosurgery," in 9th IEEE Int. Symp. on Biomedical Imaging (ISBI), pp. 1643-1646 (2012).

9. C. R. Tracy et al., "Characterisation of renal ischemia using DLP hyperspectral imaging: a pilot study comparing artery-only occlusion versus artery and vein occlusion," J. Endourol. 24(3), 321-325 (2010).

10. N. J. Crane et al., "Evidence of a heterogeneous tissue oxygenation: renal ischemia/reperfusion injury in a large animal model," J. Biomed. Opt. 18(3), 035001 (2013).

11. S. Saso et al., "Achieving uterine auto-transplantation in a sheep model using iliac vessel anastomosis: a short-term viability study," Acta Obstet. Gynecol. Scand. 94(3), 245-252 (2015).

12. S. Saso et al., "Uterine allotransplantation in a rabbit model using aortocaval anastomosis: a long-term viability study," Eur. J. Obstet. Gynecol. Reprod. Biol. 182, 185-193 (2014).

13. N. T. Clancy et al., "Multispectral imaging of organ viability during uterine transplantation surgery," Proc. SPIE 8935, 893510 (2014).

14. N. T. Clancy et al., "Registration and analysis of multispectral images acquired during uterine transplantation surgery," in OSA Biomed BSu3A.73 (2012). 
15. X. Du et al., "Robust surface tracking combining features, intensity and illumination compensation," Int. J. Comput. Assist. Radiol. Surg. 10(12), 1915-1926 (2015).

16. N. T. Clancy et al., "Multispectral image alignment using a three channel endoscope in vivo during minimally invasive surgery," Biomed. Opt. Express 3(10), 2567-2578 (2012).

17. S. A. Prahl, "Tabulated molar extinction coefficient for hemoglobin in water," http://omlc.ogi.edu/spectra/hemoglobin/summary.html (4 March 1998).

18. N. Bosschaart et al., "A literature review and novel theoretical approach on the optical properties of whole blood," Laser. Med. Sci. 29(2), 453479 (2014).

19. R. D. Shonat et al., "Near-simultaneous hemoglobin saturation and oxygen tension maps in mouse brain using an AOTF microscope," Biophys. J. 73(3), 1223-1231 (1997).

20. B. S. Sorg et al., "Hyperspectral imaging of hemoglobin saturation in tumor microvasculature and tumor hypoxia development," J. Biomed. Opt. 10(4), 044004 (2005).

21. K. Zuzak et al., "Visible reflectance hyperspectral imaging: characterization of a noninvasive, in vivo system, for determining tissue perfusion," Anal. Chem. 74(9), 2021-2028 (2002).

22. S. Wirkert et al., "Endoscopic sheffield index for unsupervised in vivo spectral band selection," in Medical Image Computing and ComputerAssisted Intervention (MICCAI)—Int. Workshop on Computer-Assisted and Robotic Endoscopy (CARE) (2014).

23. N. T. Clancy et al., "Multispectral imaging using a fast filter wheel system during vascular surgery," in Proc. of the Hamlyn Symp. on Medical Robotics, pp. 69-70 (2013).

24. S. J. Wirkert et al., "Robust near real-time estimation of physiological parameters from megapixel multispectral images with inverse Monte Carlo and random forest regression," Int. J. Comput. Assist. Radiol. Surg. 11(6), 909-917 (2016).

25. M. Brännström, "Uterus transplantation," Curr. Opin. Organ Transplant. 20(6), 621-628 (2015).

26. W. Fageeh et al., "Transplantation of the human uterus," Int. J. Gynaecol. Obstet. 76(3), 245-251 (2002).

Neil T. Clancy is an Imperial College junior research fellow at the Hamlyn Centre for Robotic Surgery and the Department of Surgery and Cancer, Imperial College London. His research interests focus on the implementation of biophotonic imaging techniques in endoscopic clinical application areas, including multi- and hyperspectral imaging, polarization, structured lighting, and illumination sources for minimally invasive surgery.

Srdjan Saso is an honorary clinical lecturer at the Institute of Reproductive and Developmental Biology, Department of Surgery and Cancer, and the Early Pregnancy and Acute Gynaecology Unit at Queen Charlotte's Hospital, Hammersmith Hospital, Imperial College London. $\mathrm{He}$ is the principal investigator in the first UK human trial in uterine transplantation.

Danail Stoyanov is a senior lecturer in the Centre for Medical Image Computing and Department of Computer Science at University College London. He leads the Surgical Robot Vision Group working on computer vision, medical image computing, medical robotics, and image-guided surgery.

Vincent Sauvage completed his $\mathrm{PhD}$ at The Hamlyn Centre, Imperial College London, under the supervision of Professor Daniel S. Elson. His work focussed on multispectral imaging of tissue oxygenation, as well as diffuse reflectance and autofluorescence spectroscopy for detection of nonalcoholic fatty liver disease. He has implemented these techniques on laparoscopic and flexible probe platforms.

David J. Corless is a consultant general and gastrointestinal surgeon based at Leighton Hospital, Mid Cheshire Hospitals NHS Foundation Trust. He has a special interest in minimally invasive surgery and the upper gastrointestinal tract. In addition to uterine transplantation, he also has research interests in clinical surgery and has published work on cholecystectomy and hernia surgery.

Michael Boyd worked as theatre manager at the Royal Veterinary College's Biological Services Unit. His research interests have included noninvasive monitoring of the uterine blood supply and investigation of changes to the vasculature during gestation in sheep.

David E. Noakes is an emeritus professor of the Royal Veterinary College, London. His research has included gastrointestinal disease in the neonatal pig and calf, as well as veterinary obstetrics and diseases of reproduction. He has published more than 130 full research papers in refereed journals and is an honorary fellow of the Royal Veterinary College.

Meen-Yau Thum is a clinical lead of the Ovum Donation Team at The Lister Hospital, London, and is a member of the advisory board of Uterine Transplantation UK. His research interests include reproductive immunology, including natural killer cells, recurrent failed implantation after IVF treatment, and recurrent miscarriage of immunological origins.

Sadaf Ghaem-Maghami is a consultant gynecological oncologist at the Institute of Reproductive and Developmental Biology, Hammersmith Hospital Campus, Imperial College London. She currently leads a group in translational research on gynecological cancers, particularly biomarker discovery and development (specifically immunological markers) in ovarian and endometrial cancers.

James Richard Smith is a consultant gynecologist and honorary senior lecturer at the West London Gynaecological Cancer Centre, Queen Charlotte's and Chelsea Hospital, Imperial College London. $\mathrm{He}$ is the leader of the UK Uterine Transplantation Research Team and has other research interests, including fertility sparing surgery for women with cancer.

Daniel S. Elson is professor of surgical imaging and biophotonics in the Hamlyn Centre for Robotic Surgery, Institute of Global Health Innovation and Department of Surgery and Cancer at St. Mary's Hospital. His research interests lie in the development and application of photonics technology to medical imaging, including multispectral imaging, ultrasound mediated optical tomography, structured lighting, light sources in endoscopy, and scattering spectroscopy. 